\title{
The influence of repetition vs. no repetition given equal presentation time on the learning of connected discourse
}

\author{
DAVID J. KING \\ State University of New York, College at Oswego, Oswego, New York 13126
}

\begin{abstract}
Subjects were required to learn a 25 words-in-length passage of connected discourse. The general design was a single $2 \times 5$ factorial. The dichotomous dimension was repetition or no repetition of the learning material. The other dimension represented total presentation time and ranged from 20 to 60 seconds. There was a lack of significance of total presentation time on total learning time but a consistent and strong superiority of the repetition condition on the no repetition condition.
\end{abstract}

The examination of the influence of temporal variables on the learning of connected discourse can be viewed from a variety of persepectives. Consider, for example, the learning of a 25 words in length passage of two sentences. One way of looking at temporal variables is to manipulate the time that each word is presented (on each trial) and/or the time between each word. If the learning material is to be presented on slides, or better, on $16-\mathrm{mm}$ film by a motion analysis projector, a convenient terminology identifies the length of time the word is presented as the exposure interval and the length of time between the words as the interitem interval. Exposure and interitem interval variations can be combined in factorial designs to assess their influence in the learning of connected discourse. When such studies are done, it is most profitable to examine them with respect to two questions. First, given that a total presentation time (TPT) can be arrived at in a number of different ways (two exposure intervals and one interitem interval yields the same TPT as one exposure interval and two interitem intervals, etc.), do these different ways of composing the TPT result in differences in total learning tiem (TLT)? A second question takes advantage of the very different TPTs that are generated in such designs (e.g., one exposure interval and zero interitem intervals yield a much shorter TPT than three exposures and three interitem intervals). In this context, the questions concern the form of the relationship between variations in TPT and consequent changes in TLT. As an aside, both of the above questions are frequently examined against the background of the total time hypothesis. The total time hypothesis can and does take on a variety of forms, as will become apparent in what follows. In the above context, the total time hypothesis might be stated as "equal amounts of learning take equal amounts of time." Considering the two questions posed above, recent evidence suggests support for the first

Requests for reprints should be sent to David J. King, Department of Psychology, State University of New York College at Oswego, Oswego, New York 13126. question (Postman \& Warren, 1972; King, 1974) and a lack of support for the second (Roberts, 1972; King, 1974). Support for the second question is often evaluated against the suggestion of Murdock (1960) although other alternatives seem possible.

Another temporal variable subject to easy manipulation is the time period between successive trials, the intertrial interval. This can be varied from very small intervals to extensive time periods. At some point as one increases the intertrial interval, the study would leave the area of consideration of "temporal variables" and merge into a study of massed vs. distributed practice. Hall (1971) has summarized this research to the effect that distributed practice has clear and strong superiority in serial learning but only a very weak advantage in paired associate learning. It would be difficult to integrate extensive variations in the intertrial interval with an analysis of the total time hypothesis. Control and/or variations over recall time is still another manipulable temporal variable. This could be done by using a paced method of recall or a fixed amount of time available for total recall. Given large individual differences in such things as writing speed, it is difficult to suggest whether or not such control would make things clearer or more confused. Still another temporal variable that is manipulable but somewhat difficult to deal with is the differential (i.e., nonequal) placement of interitem intervals throughout the learning material. In the discussion of the factorial design involving the exposure and interitem intervals in the preceding paragraph, it must hold that, for a given condition, the duration of interitem intervals must be the same. Experimentally, and probably psychologically, this need not and even should not be the case. To take but one example, there is considerable reason to suggest that the placement of "larger" interitem intervals at the border between constituent phrases (linguistic breaks) should prove easier to learn than either inappropriately placed breaks or equally dispersed interitem intervals. Initial results (King, 1973) using oral presentation lend support 
to this idea. The above temporal variables discussed in this paragraph (intertrial interval, recall interval, and nonequal placement of interitem intervals) will not be further discussed in this report (neither will a number of additional temporal variations that can be dreamed up but seem of trivial import).

The present paper will focus on still another temporal variable, that of the influence of repetition vs. no repetition given equal presentation times. In this context, one may restate the total time hypothesis in a slightly different way, namely, "a fixed amount of time is necessary to learn a fixed amount of material regardless of the number of individual trials into which that time is divided (Cooper \& Pantle, 1967, p. 221)." From this statement of the hypothesis, it is clear that our two-sentence learning material should be learned in the same amount of time whether it is presented once for $20 \mathrm{sec}$ or twice at $10 \mathrm{sec}$ each. In their review of the total time hypothesis literature, Cooper and Pantle (1967) found supportive evidence minimal in the area of serial learning. This lack of serial learning support contrasted with the more positive evidence cited for both paired-associate learning or free recall.

\section{METHOD}

The learning material consisted of a two-sentence passage of 25 words in length. The passage read: "The early morning sun shining through the yellow curtain gave the bedroom a translucent quality. George opened one eye and quickly closed it in disgust." The learning material was prepared on 16- $\mathrm{mm}$ film in accordance with the following schedule. For this study, eacn word was presented once on each frame with no blank frames intervening among the 25 words. Following these 25 frames, there was one blank frame and this sequence was repeated a second, third, fourth, fifth, and sixth time. A motion analysis projector was used so that the time per frame of the film could be adjusted as necessary. The general desing of the study was a 2 by 5 factorial. The dichotomous dimension represented either repetition of the two sentences (i.e., the subjects were exposed to the sentences two or more times) or no repetition (i.e., the subjects were exposed to the sentences only once). The other dimension represented total learning time (TLT). The exact learning times used were (in seconds) $20,30,40,50$, and 60 . For the repetition condition, the speed of the motion analysis projector was set at $.4 \mathrm{sec}$ per frame. This provided the appropriate TLTs as per the above design. For the no-repetition condition, the rate of presentation was adjusted so that one presentation of the sentences would take the appropriate amount of time. The actual design had a third dimension of sex. Five subjects, for a total of 100 subjects, were in each of the 20 cells of the design.

Following each TLT condition, the subjects were required to write the material down on a sheet of paper provided in a column, one word on each line. Subjects were run one at a time with trials continued until a perfect recall (except for spelling errors, etc.) was obtained.

The dependent variaables analyzed were the number of trials to criterion, the number of words produced on the first recall, the number of sequences (three-word sequences) recalled on the first recall, and the total learning time (the time, in seconds, from the start of the first presentation to the completion of a perfect recall).

\section{RESULTS AND DISCUSSION}

The results will emphasize the total learning time (TLT) data. The 2 by 2 by 5 ANOVA for the trials to criterion produced two significant findings. There was a significant difference between the sexes $[F(1,80)=$ $15.12, \mathrm{p}<.001]$, and another significant difference between the repetition-no repetition condition, $[F(1,80)$ $=17.01, \mathrm{p}<.001]$. The mean number of trials to criterion for males and females, respectively, were 3.0 and 2.34. The mean number of trials to criterion for the repetition-no repetition condition, respectively, were 2.32 and 3.02. A similar ANOVA on the number of words produced on the first recalled showed only one significant effect (excluding two marginal .05 effects), that for repetitions $[F(1,80)=16.29, p<.001]$. The mean number of words produced on the first recall for the repetition and no repetition conditions, respectively, were 14.92 and 9.42. The results of the ANOVA for the sequences recall on the first recall was slightly more complex with all three main effects reaching significance. For sex $[F(1,80)=8.48, p<.005]$; for TLT $[\mathrm{F}(4,80)=4.38, \mathrm{p}<.01]$, and, finally, for the repetitions effects $[F(1,80)=27.40, p<.001]$. Females were superior to males with a mean number of sequences of 13.70 as contrasted with 10.64 . The repetition condition yielded a mean of 14.92 sequences as contrasted with 9.42 for the no repetition condition. The mean number of sequences recalled as a function of TPT were, in increasing order of TLT, 8.40, 12.45, $11.95,12.95$, and 15.15 .

All three of the above dependent variables agreed on the superiority of repetitions over no repetitions. Two of the variables suggest the superiority of females over males while one suggests a significant effect on TPT.

For a study of this nature, relating as it does to such concerns as the total time hypothesis, the dependent variable of greatest import is TLT. The results of the ANOVA on the TLT data were very clear. Only two effects were significant, sex and repetitions. For sex $[F(1,80)=17.09, p<.001]$, while for repetitions $[F(1,80)=16.80, p<.001]$. The mean TLT (in seconds) was 411 for the males and 297 for the females. Similar mean TLTs for the repetitions condition was 297 and for the no repetitions, 411 .

The lack of significant effect of TPT on TLT is most important. It will be recalled that one form of the total time hypothesis suggested that a fixed amount of time necessary to learn a fixed amount of material regardless of the number of individual trials into which that time was divided. Clearly, with variations in TPT resulting in no covariations in TLT, this form of the total time hypothesis is not supported. The findings, however, do lend strength to the previous speculation that "except at the briefest of total presentation times ... the relationship between total presentation time and total 
learning time is nil (King, 1974, p. 588)." A second way of examing the total time hypothesis would be the differences between repetition and no repetition for any and all TPTs. The total time hypothesis would, of course, predict no difference between repetition and no repetition. The results clearly, strongly, and consistently show the repetition condition superior to the no repetition condition.

Several cautions should be stated to preven overgeneralization of the above results. First, and perhaps most important, the above work relates to connected discourse learning. Findings using unconnected material and other learning procedures may or may not be similar. I doubt any great similarity. Second, the preparation of the learning material on the film for this study was done by the elimination of, let alone variations in, the interitem interval. That is, as contrasted with previous studies (King, 1973, 1974), there were no blank frame(s) between the words of the sentences. The only blank frame(s) were placed between repetitions of the sentences, and only one at that. As the interitem interval, controlled by the presence of one or more blank frames, is almost certainly used for rehearsal by the learning subject, the superiority of repetition over no repetition is unknown should such variations be introduced.

\section{REFERENCES}

Cooper, E. H., \& Pantle, A. J. The total-time hypothesis is verbal learning. Psychological Bulletin, 1967, 68, 221-234.

Hall, J. F. Verbal learning and retention. Philadelphia: Lippincott, 1971.

King, D. J. Presentation time and method of reading in the learning of connected discourse. The Journal of General Psychology, 1973, 88, 283-289.

King, D. J. Total presentation time and total learning time in connected discourse learning. Journal of Experimental Psychology, 1974, 103, 586-589.

Murdock, B. B. The immediate retention of unrelated words. Journal of Experimental Psychology, 1960, 60, 222-234.

Postman, L., \& Warren, L. Test of the total-time hypothesis in free-recall learning. Journal of Experimental Psychology, 1972, 96, 176-183.

Roberts, W. A. Free recall of word lists varying in length and rate of presentation: a test of total-time hypothesis. Journal of Experimental Psychology, 1972, 92, 365-372.

(Received for publication January 25, 1975.) 